\title{
Assessment of dietary food and nutrient intake and bone density in children with eczema
}

\author{
TF Leung *, SS Wang, Flora YY Kwok, Lesley WS Leung, CM Chow, KL Hon
}

\section{A B S T R A C T}

Introduction: Dietary restrictions are common among patients with eczema, and such practice may lead to diminished bone mineral density. This study investigated dietary intake and bone mineral density in Hong Kong Chinese children with eczema.

Methods: This cross-sectional and observational study was conducted in a university-affiliated teaching hospital in Hong Kong. Chinese children aged below 18 years with physician-diagnosed eczema were recruited from our paediatric allergy and dermatology clinics over a 6-month period in 2012. Subjects with stable asthma and/or allergic rhinitis who were free of eczema and food allergy as well as non-allergic children were recruited from attendants at our out-patient clinics as a reference group. Intake of various foods and nutrients was recorded using a food frequency questionnaire that was analysed using Foodworks Professional software. Bone mineral density at the radius and the tibia was measured by quantitative ultrasound bone sonometry, and urinary cross-linked telopeptides were quantified by immunoassay and corrected for creatinine level.

Results: Overall, 114 children with eczema and 60 other children as reference group were recruited. Eczema severity of the patients was classified according to the objective SCORing Atopic Dermatitis score. Males had a higher daily energy intake than females (median, 7570 vs 6736 $\mathrm{kJ}$; $\mathrm{P}=0.035$ ), but intake of any single food item or

This article was published on 4 Aug 2017 at www.hkmj.org. nutrient did not differ between them. Compared with the reference group, children with eczema had

a higher intake of soybeans and miscellaneous dairy products and lower intake of eggs, beef, and shellfish. Children with eczema also consumed less vitamin D, calcium, and iron. The mean (standard deviation) bone mineral density Z-score of children with eczema and those in the reference group were 0.52 $(0.90)$ and $0.55(1.12)$ over the radius $(\mathrm{P}=0.889)$, and $0.02(1.03)$ and -0.01 (1.13) over the tibia $(\mathrm{P}=0.886)$, respectively. Urine telopeptide levels were similar between the groups. Calcium intake was associated with bone mineral density Z-score among children with eczema.

Conclusions: Dietary restrictions are common among Chinese children with eczema in Hong Kong, who have a lower calcium, vitamin D, and iron intake. Nonetheless, such practice is not associated with changes to bone mineral density or bone resorptive biomarker.

\section{Hong Kong Med J 2017;23:470-9}

DOI: $10.12809 / \mathrm{hkmj} 164684$

\author{
${ }^{1}$ TF Leung *, MD, FRCPCH \\ ${ }^{1}$ SS Wang, $\mathrm{PhD}$ \\ ${ }^{1}$ FYY Kwok, MPhil \\ 2 LWS Leung, BSc \\ ${ }^{1} \mathrm{CM}$ Chow, MB, ChB \\ ${ }^{1} \mathrm{KL}$ Hon, MD, FAAP
}

Department of Paediatrics, The Chinese University of Hong Kong, Prince of Wales Hospital, Shatin, Hong Kong

${ }^{2}$ Faculty of Science, The University of Melbourne, Melbourne, Victoria, Australia

*Corresponding author: tfleung@cuhk.edu.hk

New knowledge added by this study

- Hong Kong children with moderate-to-severe eczema had a lower consumption of eggs, beef, and shellfish as well as vitamin $\mathrm{D}$, calcium, and iron.

- Children with eczema had an increased intake of soybean and miscellaneous dairy products.

- These changes in dietary and nutrient intake were not associated with altered bone mineral density or urinary levels of cross-linked $\mathrm{N}$-telopeptides of type 1 collagen in our children with eczema.

Implications for clinical practice or policy

- Nutritional assessment and counselling should be offered to parents with children who have moderate-tosevere eczema.

- Children with eczema who have extensive food avoidance or impaired growth should undergo allergy evaluation so that their family can follow evidence-based advice about dietary modification.

- Bone mineral density assessment is unnecessary for the majority of children with eczema.

\section{Introduction}

Eczema is a chronic inflammatory skin disease associated with cutaneous hyperreactivity to environmental stimuli such as microbial exposure and food ingestion that are otherwise tolerated by unaffected subjects. ${ }^{1}$ In our community study, nearly 
one third of preschool children had eczema and 8.1\% had parent-reported adverse food reactions. ${ }^{2}$ Thus, there is a significant health care burden from both eczema and food allergy in Hong Kong. Food allergy is believed by many patients and their family to be a major cause of eczema. Many traditional Chinese medicine practitioners also advise extensive food avoidance for eczema. Our previous study reported that over half of local children with eczema practised food avoidance. $^{3}$ There are, however, limited objective data on the intake of specific food items and nutrients in children with eczema. Henderson and Hayes ${ }^{4}$ reported the correlation between dietary calcium intake and bone mineral density (BMD) in 55 children and adolescents aged 5 to 14 years with cow's milk sensitisation. Their calcium intake was determined using a food frequency questionnaire (FFQ). The bone density Z-score of their patients with a milk allergy serially increased with increasing calcium intake. In another study, Jensen et $\mathrm{al}^{5}$ investigated $\mathrm{BMD}$ in children aged 8 to 17 years with verified cow's milk allergy (CMA) for more than 4 years and compared them with 343 healthy controls. Their patient's BMD was markedly reduced for age, and height for age was reduced indicating 'short' bones. Furthermore, calcium consumption was about $25 \%$ of that recommended. In another study, BMD was assessed in 27 young children with CMA. ${ }^{6}$ During bone resorption, osteoclasts secrete a mixture of acid and neutral proteases that degrade the collagen fibrils into molecular fragments including $\mathrm{C}$-terminal telopeptide. Its aspartic acid changes from alpha to beta form as bone ages. The latter form called beta-crosslaps is a specific marker for bone resorption; its concentration was lower in CMA patients than in controls, indicating an increased bone turnover in the former. Ten CMA patients had a BMD Z-score of less than -1 standard deviation (SD) value. Despite these results, there was limited evidence of compromised bone health in eczema patients when such results were adjusted for confounders such as calcium intake and physical activity. This study investigated intake of food items and nutrients as well as BMD in Chinese children in Hong Kong with varying degrees of eczema, and compared these values with unaffected children.

\section{Methods}

\section{Study population}

This cross-sectional study recruited ethnic Chinese children aged below 18 years with physiciandiagnosed eczema from our paediatric allergy and dermatology clinics over a 6-month period in 2012. Eczema was diagnosed using standard criteria, ${ }^{7}$ and disease severity was assessed by SCORing Atopic Dermatitis. $^{8}$ Patients were treated with topical mometasone furoate only. Those prescribed other

\section{評估兒童濕疹患者的食物和營養攝取量與 其骨密度}

梁廷勳、王术欣、郭燕瑩、梁韻生、周中武、韓錦倫

引言：飲食禁忌在濕疹患者中很普遍, 這可能導致骨質密度降低。本 研究探討香港華籍兒童濕疹患者的飲食和其骨質密度的關係。

方法：這項橫斷面觀察研究在香港一所大學附屬教學醫院內進 行。2012年的6個月內在本院的兒科過敏和皮膚科診所招募了 18 歲以 下的兒童濕疹患者。亦在本院的門診診所招募了沒有過敏的兒童以及 具有穩定的哮喘狀況和/或過敏性鼻炎但並沒有濕疹和食物過敏的兒童 作為參考組。使用一份食物頻率問卷並透過Foodworks Professional軟 件記錄及分析參與者對不同食物和營養的攝取。利用定量超聲骨測量 法量度半徑和脛骨的骨質密度, 並通過免疫測試對尿交聯氨基端肽進 行定量並校正肌酐水平。

結果：研究期間共招募了 114 例兒童濕疹患者和 60 例參考組別的 兒童。按「異位性皮膚炎評分」（SCORAD）評估他們的濕 疹情況。男性每日能量攝取量高於女性（中位數, $7570 \mathrm{~kJ}$ 比 $6736 \mathrm{~kJ} ; \mathrm{P}=0.035$ ），但兩個性別之間對攝入任何單一食物或營養成 分則沒有差異。與參考組別比較, 濕疹患者的大豆和奶類製品攝取量 較高, 但雞蛋、牛肉和貝殼類的攝取量較低。濕疹患者亦攝取較少維 生素D、鉦和鐵。濕疹患者和參考組別的平均 (標準差) 骨質密度Z評 分方面, 半徑分別為0.52 (0.90) 和0.55 ( 1.12 )〔 $\mathrm{P}=0.889 〕$, 而 脛骨分別為0.02（1.03）和-0.01（1.13）〔 $\mathrm{P}=0.886 〕 。$ 兩組之間尿 肽水平相若。䤮攝取量與濕疹患者的骨質密度Z評分相關。

結論：飲食禁忌在香港華籍兒童濕疹患者中很普遍，他們的銔、維生 素D和鐵攝取量較低。儘管如此, 這對骨質密度或骨再生指標的變化 無關。

topical steroids were changed to this drug for at least 4 weeks before the study. Patients prescribed oral immunosuppressive drugs within 6 months were excluded. Subjects with stable asthma and/ or allergic rhinitis who were free of eczema and food allergy as well as non-allergic children were recruited from attendants at our out-patient clinics as a reference group. As an inclusion criterion, subjects in the reference group had no dietary restrictions. Following informed written consent, our research staff recorded subjects' intake of a wide spectrum of dietary components using a Chinese FFQ. Children who attended secondary school and beyond were assessed using the adolescent/adult version, ${ }^{9,10}$ whereas those in primary schools and below were assessed using the preschool version. ${ }^{11}$ Intake of individual food items and groups of major nutrients as recorded in FFQ were quantified and analysed by Foodworks Professional software (version 7; Xyris, Brisbane, Australia). This software included only certain Chinese food items commonly found in Australia. Thus, our co-author (FYYK), who has a dietetic qualification, added new Chinese food data with reference to a comprehensive Chinese food composition database published in 2004 by 
the Peking University Medical Press (available upon request). These new food items were saved in the software as a new food composition database so that their nutrient data could be computed. Physical activity level of participants was assessed using a Physical Self-Description Questionnaire. ${ }^{12}$ The study was approved by the Clinical Research Ethics Committee of our university. All participants provided written informed consent.

\section{Urinary concentration of cross-linked $\mathrm{N}$-telopeptides of type 1 collagen}

Urinary concentrations of cross-linked Ntelopeptides of type 1 collagen (NTx), biomarkers of bone resorption, ${ }^{13}$ were measured by enzyme-linked immunosorbent assay (Wampole Laboratories, Princeton [NJ], US) with a lower limit of detection set at $20 \mathrm{nM}$ of bone collagen equivalent. Results were corrected for creatinine that was measured by modified Jaffe reaction (Roche Diagnostics $\mathrm{GmbH}$, Mannheim, Germany).

\section{Bone mineral density measurement}

Participants' BMD was measured at the mid-point of the radius in the non-dominant arm and at the left tibia using quantitative ultrasound bone sonometry (QUBS) to determine the velocity of ultrasound wave, expressed as the speed of sound in $\mathrm{m} / \mathrm{s}$, using Omnisense 7000P (BeamMed, Petach Tikva, Israel) as described previously. ${ }^{14,15}$ This machine compared speed of sound measurements to a built-in reference database of a healthy urban Chinese population of
797 boys and 760 girls aged 0 to 18 years. As described in the manufacturer's manual, subjects with previous osteoporotic fractures, use of medications affecting bone health, presence of a disease known to affect bone metabolism, recent prolonged immobilisation, or a systemic malignant disease within 5 years were excluded from such reference database. The Omnisense devices were transported from place to place, and the same group of operators performed all measurements. Results of BMD were then expressed as age- and gender-matched Z-score.

\section{Statistical analysis}

Dietary food and nutrient intake, BMD Z-scores, and urine NTx levels between different groups were analysed by Student's $t$ test or analysis of variance (ANOVA). The relationship between eczema and dietary, BMD, and urinary variables that differed significantly between children with eczema and those in the reference group were confirmed by multivariable stepwise binary logistic regression adjusted for age, gender, body mass index (BMI), physical activity level, and co-morbid allergic diseases as covariates. The correlations between clinical variables, BMD Z-scores, and urine NTx levels were analysed by Pearson correlation. Continuous variables with skewed data distribution were transformed to achieve normal distribution prior to analyses. All analyses were performed with the use of SPSS (Windows version 18.0; SPSS Inc, Chicago [IL], US). The level of significance was set at 0.05 , and all P values were two-tailed.

TABLE I. Clinical characteristics of 174 study participants

\begin{tabular}{|c|c|c|c|c|}
\hline & \multicolumn{3}{|c|}{ Mean \pm standard deviation or No. (\%) } & \multirow[t]{2}{*}{$P$ value } \\
\hline & $\begin{array}{l}\text { Reference group* } \\
(n=60)\end{array}$ & Mild eczema $(n=64)$ & $\begin{array}{l}\text { Moderate-to-severe } \\
\text { eczema }(n=50)\end{array}$ & \\
\hline Age (years) & $10.0 \pm 5.0$ & $9.8 \pm 5.4$ & $11.9 \pm 3.8$ & 0.050 \\
\hline Male & $35(58.3)$ & $27(42.2)$ & $27(54.0)$ & 0.177 \\
\hline Body weight (kg) & $34.9 \pm 19.9$ & $34.2 \pm 20.2$ & $40.9 \pm 17.0$ & 0.141 \\
\hline Standing height $(\mathrm{cm})$ & $133.3 \pm 28.9$ & $131.3 \pm 28.5$ & $142.6 \pm 21.8$ & 0.070 \\
\hline BMI $\left(\mathrm{kg} / \mathrm{m}^{2}\right)$ & $17.7 \pm 4.3$ & $17.7 \pm 3.8$ & $19.1 \pm 4.0$ & 0.144 \\
\hline BMI Z-score & $-0.10 \pm 1.28$ & $0.03 \pm 1.20$ & $0.35 \pm 0.97$ & 0.128 \\
\hline Physical activity level ${ }^{12}$ & & & & 0.032 \\
\hline Light & $12(20.0)$ & $25(39.1)$ & $20(40.0)$ & \\
\hline Moderate & $37(61.7)$ & $23(35.9)$ & $19(38.0)$ & \\
\hline Heavy & $11(18.3)$ & $17(26.6)$ & $11(22.0)$ & \\
\hline Co-morbid asthma* & $3(5.0)$ & $12(18.8)$ & $17(34.0)$ & $<0.001$ \\
\hline Co-morbid allergic rhinitis* & $13(21.7)$ & $30(46.9)$ & $27(54.0)$ & 0.001 \\
\hline
\end{tabular}

Abbreviation: $\mathrm{BMI}=$ body mass index

* Reference subjects with these co-morbid allergies must not practise any dietary restriction and also be free from any inhaled or intranasal corticosteroid treatment

$\dagger$ Analysed by one-way analysis of variance for numerical variables and $\chi^{2}$ test for categorical variables 


\section{Results}

A total of 114 children with eczema and other 60 children as the reference group were recruited (Table 1 ). The means ( \pm SDs) age of children in the reference group, children with mild eczema, and children with moderate-to-severe eczema were $10.0 \pm 5.0,9.8 \pm$ 5.4 , and $11.9 \pm 3.8$ years, respectively. Age, gender, and anthropometric variables did not differ among children with eczema and those in the reference group. Table 2 describes the children's daily food intake adjusted for total energy as recorded by FFQ. Males had a higher daily energy intake than females (median [interquartile range]: 7570 [6267-9487] kJ vs 6736 [5438-8547] kJ; P=0.035), but intake of any single food item or nutrient did not differ when adjusted for daily energy intake. Multivariable stepwise binary logistic regression analyses revealed that a higher intake of soybeans and soybean products as well as miscellaneous dairy products was associated with an increased risk of eczema, although statistically significant associations were only found between the third tertile of soybean intake and mild eczema, as well as between the second tertile and mild eczema, and between the third tertile and moderate-to-severe eczema for intake of miscellaneous dairy products. A higher consumption of eggs, beef, and shellfish was associated with lower risk of eczema (Table 3). Children with eczema and those in the reference group consumed similar supplements of cod liver oil, fish oil, vitamins, and calcium ( $\mathrm{P}>0.9$ for all; data not shown). Table 4 summarises their nutrient intake. Patients with moderate-to-severe eczema consumed a lower amount of vitamin D, calcium, and iron when compared with those with mild eczema and/or those in the reference group. Children with the highest tertile of intake for vitamin $\mathrm{D}$ (odds ratio $[\mathrm{OR}]=0.16$; 95\% confidence interval $[\mathrm{CI}]$ 0.05-0.48; $\mathrm{P}=0.001)$ and calcium $(\mathrm{OR}=0.17 ; 95 \% \mathrm{CI}, 0.06-0.51 ; \mathrm{P}=0.002)$ had a lower risk for moderate-to-severe eczema than those with the lowest tertile when adjusted for age, gender, BMI Z-score, and physical activity level as covariates.

The mean BMD Z-score for children with eczema and those in the reference group was 0.52

TABLE 2. Daily food intake in 174 children with and without eczema

\begin{tabular}{|c|c|c|c|c|c|c|c|}
\hline \multirow[t]{2}{*}{ Food intake } & \multicolumn{3}{|c|}{ Median (IQR) of daily intake (g/MJ) } & \multirow[t]{2}{*}{ P value* } & \multicolumn{3}{|c|}{$P$ value between groups $†$} \\
\hline & $\begin{array}{l}\text { Reference group } \\
(n=60)\end{array}$ & ME $(n=64)$ & MSE $(n=50)$ & & $\begin{array}{l}\text { Reference } \\
\text { group vs } \\
\text { ME }\end{array}$ & $\begin{array}{l}\text { Reference } \\
\text { group vs } \\
\text { MSE }\end{array}$ & $\begin{array}{l}\text { ME vs } \\
\text { MSE }\end{array}$ \\
\hline $\begin{array}{l}\text { Bread, cereals, pasta, rice, and } \\
\text { noodles }\end{array}$ & $63.1(50.3-85.5)$ & $59.4(42.2-74.6)$ & $63.3(48.7-79.3)$ & 0.242 & - & - & - \\
\hline Fruits & $21.9(15.3-35.4)$ & $21.6(12.2-37.3)$ & $23.8(13.4-40.1)$ & 0.953 & - & - & - \\
\hline Vegetables & $25.5(15.2-40.5)$ & $20.8(12.5-40.3)$ & $27.8(19.0-47.2)$ & 0.441 & - & - & - \\
\hline Legumes & & & & & - & - & - \\
\hline Soybeans and soybean products & $5.67(1.67-12.4)$ & $7.18(4.21-18.4)$ & $7.11(2.26-16.4)$ & 0.100 & & & \\
\hline Peanuts & $0.04(0-0.53)$ & $0(0-0.26)$ & $0(0-0.24)$ & 0.248 & & & \\
\hline Tree nuts & $0(0-0.05)$ & $0(0-0.05)$ & $0(0-0.13)$ & 0.632 & & & \\
\hline Egg & $1.75(0.86-3.65)$ & $1.21(0.41-2.07)$ & $1.19(0.24-2.52)$ & 0.034 & 0.014 & 0.047 & 0.950 \\
\hline \multicolumn{8}{|l|}{ Meat } \\
\hline Pork & $3.33(1.90-5.90)$ & $3.80(1.76-7.40)$ & $5.10(2.60-10.93)$ & 0.050 & 0.726 & 0.018 & 0.046 \\
\hline Beef & $0.59(0-1.65)$ & $0.66(0.07-1.48)$ & $0.05(0-1.43)$ & 0.068 & & & \\
\hline Chicken & $3.02(2.04-5.27)$ & $3.17(1.84-5.96)$ & $3.19(1.40-5.13)$ & 0.553 & & & \\
\hline Processed meat & $1.23(0.44-2.11)$ & $1.54(0.40-3.10)$ & $1.98(0.62-3.43)$ & 0.159 & & & \\
\hline Fish/seafood & & & & & - & - & - \\
\hline Non-shellfish & $3.17(1.46-7.35)$ & $3.68(2.39-6.13)$ & $3.13(1.63-5.61)$ & 0.839 & & & \\
\hline Shellfish & $0.21(0-0.95)$ & $0.12(0-0.94)$ & $0.07(0-0.39)$ & 0.245 & & & \\
\hline \multicolumn{8}{|l|}{ Milk and dairy products $\ddagger$} \\
\hline Cow's milk & $14.90(5.66-28.00)$ & $10.10(2.48-22.20)$ & $8.36(3.51-17.50)$ & 0.079 & & & \\
\hline Miscellaneous dairy products & $0.47(0-1.85)$ & $1.30(0.33-2.63)$ & $1.66(0.40-3.29)$ & 0.013 & 0.015 & 0.007 & 0.517 \\
\hline
\end{tabular}

Abbreviations: $I Q R$ = interquartile range; $M E$ = mild eczema; $M S E$ = moderate-to-severe eczema

* Analysed by Kruskal-Wallis test for trend across the three groups

† Performed post-hoc analyses by Mann-Whitney $U$ test only for food items with statistically significant trend

‡ Cow's milk included both liquid and powder forms, whereas miscellaneous dairy products included all other processed cow's milk products such as cheese, yogurt, and ice cream 
TABLE 3. Multivariable binary logistic analyses for the associations between eczema severity and food intake*

\begin{tabular}{|c|c|c|c|c|c|c|c|c|}
\hline \multirow[t]{2}{*}{ Food intake } & \multirow{2}{*}{$\begin{array}{c}\begin{array}{c}\text { Reference } \\
\text { group } \\
(\mathrm{n}=60)\end{array} \\
\text { Ref }\end{array}$} & \multicolumn{2}{|c|}{ Mild eczema $(n=64)$} & \multicolumn{2}{|c|}{$\begin{array}{l}\text { Moderate-to-severe } \\
\text { eczema }(n=50)\end{array}$} & \multirow{2}{*}{$\begin{array}{c}\begin{array}{c}\text { Mild } \\
\text { eczema } \\
(n=64)\end{array} \\
\text { Ref }\end{array}$} & \multicolumn{2}{|c|}{$\begin{array}{l}\text { Moderate-to-severe } \\
\text { eczema }(n=50)\end{array}$} \\
\hline & & $P$ value & $\begin{array}{l}\text { Adjusted OR } \\
(95 \% \mathrm{Cl}) \dagger\end{array}$ & P value & $\begin{array}{l}\text { Adjusted OR } \\
(95 \% \mathrm{Cl}) \dagger\end{array}$ & & $P$ value & $\begin{array}{c}\text { Adjusted OR } \\
(95 \% \mathrm{Cl}) \dagger\end{array}$ \\
\hline \multicolumn{9}{|c|}{ Bread, cereals, pasta, rice, and noodles } \\
\hline Second tertile & 1.0 & 0.436 & $0.69(0.28-1.73)$ & 0.766 & $0.85(0.30-2.42)$ & 1.0 & 0.926 & $1.05(0.39-2.84)$ \\
\hline Third tertile & 1.0 & 0.223 & $0.57(0.23-1.41)$ & 0.629 & $0.78(0.28-2.16)$ & 1.0 & 0.504 & $1.42(0.51-3.92)$ \\
\hline \multicolumn{9}{|l|}{ Fruits } \\
\hline Second tertile & 1.0 & 0.106 & $0.48(0.20-1.17)$ & 0.445 & $0.68(0.25-1.83)$ & 1.0 & 0.614 & $1.28(0.49-3.39)$ \\
\hline Third tertile & 1.0 & 0.552 & $0.76(0.31-1.88)$ & 0.980 & $1.01(0.38-2.71)$ & 1.0 & 0.595 & $1.29(0.50-3.32)$ \\
\hline \multicolumn{9}{|l|}{ Vegetables } \\
\hline Second tertile & 1.0 & 0.055 & $0.41(0.17-1.02)$ & 0.745 & $1.18(0.43-3.28)$ & 1.0 & 0.074 & $2.53(0.91-6.99)$ \\
\hline Third tertile & 1.0 & 0.596 & $0.78(0.31-1.96)$ & 0.463 & $1.47(0.53-4.13)$ & 1.0 & 0.184 & $1.97(0.73-5.33)$ \\
\hline \multicolumn{9}{|c|}{ Soybeans and soybean products } \\
\hline Second tertile & 1.0 & 0.173 & $1.88(0.76-4.63)$ & 0.437 & $1.47(0.56-3.85)$ & 1.0 & 0.362 & $0.63(0.23-1.71)$ \\
\hline Third tertile & 1.0 & 0.041 & $2.59(1.04-6.43)$ & 0.169 & $1.98(0.75-5.23)$ & 1.0 & 0.505 & $0.72(0.27-1.90)$ \\
\hline \multicolumn{9}{|l|}{ Peanuts } \\
\hline Intake below median & 1.0 & 0.190 & $1.88(0.73-4.83)$ & 0.540 & $1.36(0.51-3.66)$ & 1.0 & 0.224 & $0.56(0.22-1.42)$ \\
\hline Intake above median & 1.0 & 0.473 & $0.72(0.30-1.76)$ & 0.169 & $0.51(0.20-1.33)$ & 1.0 & 0.598 & $0.76(0.27-2.11)$ \\
\hline \multicolumn{9}{|l|}{ Eggs } \\
\hline Second tertile & 1.0 & 0.099 & $0.46(0.18-1.16)$ & 0.110 & $0.43(0.15-1.21)$ & 1.0 & 0.613 & $1.28(0.50-3.27)$ \\
\hline Third tertile & 1.0 & 0.037 & $0.37(0.15-0.94)$ & 0.027 & $0.29(0.10-0.87)$ & 1.0 & 0.545 & $1.34(0.52-3.49)$ \\
\hline \multicolumn{9}{|l|}{ Pork } \\
\hline Second tertile & 1.0 & 0.666 & $0.83(0.35-1.97)$ & 0.343 & $1.62(0.60-4.39)$ & 1.0 & 0.213 & $1.90(0.69-5.20)$ \\
\hline Third tertile & 1.0 & 0.426 & $1.44(0.59-3.56)$ & 0.056 & $2.70(0.97-7.48)$ & 1.0 & 0.125 & $2.14(0.81-5.64)$ \\
\hline \multicolumn{9}{|l|}{ Beef } \\
\hline Second tertile & 1.0 & 0.262 & $1.69(0.67-4.26)$ & 0.042 & $0.34(0.12-0.96)$ & 1.0 & 0.002 & $0.20(0.07-0.54)$ \\
\hline Third tertile & 1.0 & 0.972 & $0.98(0.38-2.58)$ & 0.062 & $0.41(0.16-1.05)$ & 1.0 & 0.184 & $0.51(0.19-1.38)$ \\
\hline \multicolumn{9}{|l|}{ Chicken } \\
\hline Second tertile & 1.0 & 0.704 & $0.84(0.35-2.04)$ & 0.380 & $0.64(0.24-1.73)$ & 1.0 & 0.940 & $0.96(0.36-2.56)$ \\
\hline Third tertile & 1.0 & 0.669 & $1.22(0.49-3.03)$ & 0.631 & $1.28(0.47-3.44)$ & 1.0 & 0.868 & $1.08(0.42-2.80)$ \\
\hline \multicolumn{9}{|l|}{ Processed meat } \\
\hline Second tertile & 1.0 & 0.375 & $0.67(0.28-1.62)$ & 0.547 & $0.74(0.27-2.00)$ & 1.0 & 0.544 & $0.73(0.26-2.02)$ \\
\hline Third tertile & 1.0 & 0.145 & $2.02(0.79-5.21)$ & 0.112 & $2.27(0.83-6.26)$ & 1.0 & 0.994 & $1.00(0.39-2.57)$ \\
\hline \multicolumn{9}{|l|}{ Non-shellfish } \\
\hline Second tertile & 1.0 & 0.069 & $2.35(0.94-5.88)$ & 0.592 & $1.32(0.48-3.60)$ & 1.0 & 0.061 & $0.40(0.15-1.04)$ \\
\hline Third tertile & 1.0 & 0.422 & $1.45(0.58-3.62)$ & 0.871 & $0.93(0.36-2.36)$ & 1.0 & 0.155 & $0.49(0.18-1.31)$ \\
\hline \multicolumn{9}{|l|}{ Shellfish } \\
\hline Second tertile & 1.0 & 0.930 & $0.96(0.37-2.48)$ & 0.746 & $0.85(0.33-2.24)$ & 1.0 & 0.493 & $0.72(0.28-1.83)$ \\
\hline Third tertile & 1.0 & 0.451 & $0.70(0.28-1.76)$ & 0.016 & $0.28(0.10-0.79)$ & 1.0 & 0.027 & $0.30(0.11-0.87)$ \\
\hline \multicolumn{9}{|l|}{ Cow's milk } \\
\hline Second tertile & 1.0 & 0.102 & $0.45(0.18-1.17)$ & 0.379 & $0.65(0.24-1.71)$ & 1.0 & 0.474 & $1.40(0.56-3.51)$ \\
\hline Third tertile & 1.0 & 0.165 & $0.51(0.19-1.32)$ & 0.169 & $0.46(0.15-1.40)$ & 1.0 & 0.824 & $0.89(0.33-2.43)$ \\
\hline \multicolumn{9}{|c|}{ Miscellaneous dairy products } \\
\hline Second tertile & 1.0 & 0.010 & $3.32(1.34-8.24)$ & 0.084 & $2.52(0.88-7.20)$ & 1.0 & 0.450 & $0.67(0.23-1.91)$ \\
\hline Third tertile & 1.0 & 0.100 & $2.19(0.86-5.56)$ & 0.007 & $3.93(1.44-10.72)$ & 1.0 & 0.377 & $1.58(0.57-4.35)$ \\
\hline
\end{tabular}

Abbreviations: $\mathrm{Cl}=$ confidence interval; $\mathrm{OR}=$ odds ratio

* Dietary intakes of all subjects were grouped into tertiles

† Adjusted for age, sex, body mass index Z-score, and physical activity level 
TABLE 4. Daily nutrient intake in 174 children with and without eczema

\begin{tabular}{|c|c|c|c|c|c|c|c|}
\hline \multirow[t]{2}{*}{ Daily nutrient intake } & \multicolumn{3}{|c|}{$\begin{array}{c}\text { Median (IQR) } \\
\text { No. (\%) of children with actual intakes <DRI* }\end{array}$} & \multirow[t]{2}{*}{$P$ valuet } & \multicolumn{3}{|c|}{ P value between groups $¥$} \\
\hline & $\begin{array}{l}\text { Reference group } \\
(n=60)\end{array}$ & $M E(n=64)$ & MSE $(n=50)$ & & $\begin{array}{l}\text { Reference } \\
\text { group vs } \\
\text { ME }\end{array}$ & $\begin{array}{l}\text { Reference } \\
\text { group vs } \\
\text { MSE }\end{array}$ & $\begin{array}{l}\text { ME vs } \\
\text { MSE }\end{array}$ \\
\hline Energy & & & & & - & - & - \\
\hline in $\mathrm{kJ}$ & 7023 (5345-8496) & 7154 (5656-9054) & 7518 (6334-9488) & 0.108 & & & \\
\hline in $\mathrm{kCal}$ & $\begin{array}{l}1678(1277-2030) \\
41(68.3)\end{array}$ & $\begin{array}{c}1709(1351-2163) \\
38(59.4)\end{array}$ & $\begin{array}{c}1796(1513-2267) \\
33(66.0)\end{array}$ & 0.108 & & & \\
\hline Protein (\% of energy) & $\begin{array}{l}16.6(14.8-20.8) \\
27(45.0)\end{array}$ & $\begin{array}{l}17.2(14.2-19.5) \\
24(37.5)\end{array}$ & $\begin{array}{l}17.3(15.1-20.4) \\
16(32.0)\end{array}$ & 0.864 & - & - & - \\
\hline Fats (\% of energy) & $28.5(27.0-31.4)$ & $28.9(26.3-32.3)$ & $27.9(24.5-34.4)$ & 0.824 & - & - & - \\
\hline Saturated fat (\%) & $8.45(6.49-10.27)$ & $8.63(6.91-10.47)$ & $8.81(7.56-10.39)$ & 0.621 & - & - & - \\
\hline Polyunsaturated fat (\%) & $5.41(4.34-7.15)$ & $5.79(4.74-7.69)$ & $5.82(4.60-7.39)$ & 0.458 & - & - & - \\
\hline Carbohydrates (\% of energy) & $49.5(44.6-53.5)$ & $50.0(45.3-53.1)$ & $49.3(43.3-53.8)$ & 0.912 & - & - & - \\
\hline Vitamin A ( $\mu \mathrm{gRE} / \mathrm{MJ})$ & $\begin{array}{l}105.1(67.4-153.8) \\
27(45.0)\end{array}$ & $\begin{array}{l}91.5(73.3-136.8) \\
26(40.6)\end{array}$ & $\begin{array}{l}90.5(65.4-125.0) \\
22(44.0)\end{array}$ & 0.473 & - & - & - \\
\hline Vitamin C (mg/MJ) & $\begin{array}{l}17.6(11.9-26.8) \\
13(21.7)\end{array}$ & $\begin{array}{l}17.1(10.4-25.4) \\
\quad 19(29.7)\end{array}$ & $\begin{array}{l}15.0(10.6-21.8) \\
15(30.0)\end{array}$ & 0.350 & - & - & - \\
\hline Vitamin D ( $\mu \mathrm{g} / \mathrm{MJ})$ & $\begin{array}{l}0.27(0.10-0.61) \\
59(98.3)\end{array}$ & $\begin{array}{l}0.14(0.06-0.35) \\
\quad 62(96.9)\end{array}$ & $\begin{array}{l}0.10(0.06-0.17) \\
50(100)\end{array}$ & 0.001 & 0.037 & $<0.001$ & 0.081 \\
\hline Vitamin E (mg/MJ) & $\begin{array}{l}0.95(0.66-1.47) \\
\quad 37(61.7)\end{array}$ & $\begin{array}{l}1.01(0.66-1.50) \\
\quad 31(48.4)\end{array}$ & $\begin{array}{l}0.81(0.60-1.23) \\
\quad 33(66.0)\end{array}$ & 0.134 & & & \\
\hline Calcium (mg/MJ) & $\begin{array}{l}89.5(62.0-125.4) \\
\quad 41(68.3)\end{array}$ & $\begin{array}{l}74.4(58.8-108.8) \\
48(75.0)\end{array}$ & $\begin{array}{l}68.1(52.9-77.1) \\
\quad 42(84.0)\end{array}$ & 0.001 & 0.175 & $<0.001$ & 0.019 \\
\hline Iron (mg/MJ) & $\begin{array}{l}1.63(1.30-2.12) \\
40(66.6)\end{array}$ & $\begin{array}{l}1.59(1.24-2.08) \\
45(70.3)\end{array}$ & $\begin{array}{l}1.41(1.17-1.71) \\
\quad 38(76.0)\end{array}$ & 0.047 & 0.586 & 0.015 & 0.070 \\
\hline Zinc (mg/MJ) & $\begin{array}{l}1.41(1.18-1.65) \\
45(75.0)\end{array}$ & $\begin{array}{l}1.31(1.16-1.52) \\
49(76.6)\end{array}$ & $\begin{array}{l}1.29(1.11-1.47) \\
42(84.0)\end{array}$ & 0.094 & & & \\
\hline
\end{tabular}

Abbreviations: $\mathrm{DRI}=$ dietary reference intake; $\mathrm{IQR}$ = interquartile range; $\mathrm{ME}=$ mild eczema; $\mathrm{MJ}$ = megajoule; $\mathrm{MSE}=$ moderate-to-severe eczema; $\mathrm{RE}=$ retinol equivalents

* Age- and sex-specific DRI for Chinese, based on recommended nutrient intake for energy; protein; vitamin A, C, and D; zinc; and adequate intake for vitamin E, calcium, and iron

† Comparisons of median nutrient intake by Kruskal-Wallis test; comparisons of \% DRI of nutrient intakes by $\chi^{2}$

$\ddagger$ Pairwise comparisons of median nutrient intake by Mann-Whitney $U$ test; paired comparisons of $\%$ DRI of nutrient intakes by Yates' corrected $\chi^{2}$

TABLE 5. Differences in BMD variables between children with and without eczema

\begin{tabular}{|c|c|c|c|}
\hline & \multicolumn{2}{|c|}{$\begin{array}{c}\text { Mean (SD) } \\
\text { No. (\%) with Z-score <-1 }\end{array}$} & \multirow[t]{2}{*}{ P value* } \\
\hline & Reference group $(n=60)$ & Eczema $(n=114)$ & \\
\hline \multicolumn{4}{|l|}{ BMD Z-score } \\
\hline Radius & $\begin{array}{r}0.55(1.12) \\
6(10.0)\end{array}$ & $\begin{array}{l}0.52(0.90) \\
5(4.4)\end{array}$ & $\begin{array}{l}0.889 \\
0.148\end{array}$ \\
\hline Tibia & $\begin{array}{r}-0.01(1.13) \\
10(16.7)\end{array}$ & $\begin{array}{r}0.02(1.03) \\
18(15.8)\end{array}$ & $\begin{array}{l}0.886 \\
0.881\end{array}$ \\
\hline Urine NTx-to-creatinine ratio (nM/mM) & $339(174)$ & $313(223)$ & 0.451 \\
\hline
\end{tabular}

Abbreviations: $\mathrm{BMD}=$ bone mineral density; $\mathrm{NTx}$ = cross-linked N-telopeptides of type I collagen; $\mathrm{SD}=$ standard deviation

* Analysed by Student's $t$ test for means and $\chi^{2}$ for numbers

and 0.55 over the radius $(\mathrm{P}=0.889)$, and 0.02 and $(\mathrm{P}=0.296$ and 0.661 , respectively). The BMD Z-score -0.01 over the tibia $(\mathrm{P}=0.886)$ [Table 5]. The BMD at the tibia correlated inversely with children's did not differ between children with mild and age $(r=-0.282, \mathrm{P}<0.001)$, but the Z-score at both moderate-to-severe eczema over these two regions regions was independent of BMI Z-score. Age of 
TABLE 6. Associations between daily calcium intake and BMD and urine NTx among children with and without eczema

\begin{tabular}{|c|c|c|c|c|c|c|c|}
\hline & \multicolumn{3}{|c|}{ Daily calcium intake } & \multirow[t]{2}{*}{$P$ value } & \multicolumn{3}{|c|}{ Post-hoc comparison*, mean difference (SE) } \\
\hline & First tertile & Second tertile & Third tertile & & 1st vs 2nd & 1st vs 3rd & 2nd vs 3rd \\
\hline \multicolumn{8}{|l|}{ Reference subjects $(n=60)$} \\
\hline Radius Z-score & $0.46(1.16)$ & $0.52(1.15)$ & $0.66(1.11)$ & 0.850 & & & \\
\hline Tibia Z-score & $0.01(1.22)$ & $-0.15(1.33)$ & $0.12(0.84)$ & 0.764 & & & \\
\hline Urine NTx-to-Cr ratio (nM/mM) & $251(152)$ & $400(185)$ & $375(156)$ & 0.018 & $-150(55) \dagger$ & $-124(54)$ & $26(56)$ \\
\hline \multicolumn{8}{|l|}{ With eczema $(n=114)$} \\
\hline Radius Z-score & $0.72(0.80)$ & $0.18(0.72)$ & $0.67(1.08)$ & 0.016 & $0.53(0.20) \dagger$ & $0.04(0.20)$ & $-0.50(0.20) \dagger$ \\
\hline Tibia Z-score & $-0.09(1.06)$ & $-0.25(1.04)$ & $0.39(0.87)$ & 0.015 & $0.16(0.23)$ & $-0.49(0.23)$ & $-0.65(0.23) \dagger$ \\
\hline Urine NTx-to-Cr ratio (nM/mM) & $347(226)$ & $325(255)$ & $268(183)$ & 0.312 & & & \\
\hline
\end{tabular}

Abbreviations: BMD = bone mineral density; $\mathrm{Cr}=$ creatinine; NTx = cross-linked $\mathrm{N}$-telopeptides of type I collagen; SE = standard error

* Performed only for variables with significant results by one-way analysis of variance, and analysed with Bonferroni correction for multiple comparisons

$+\mathrm{P}<0.05$

onset of eczema did not influence BMD Z-score at the radius $(\mathrm{P}=0.349)$ or tibia $(\mathrm{P}=0.240)$, nor was it associated with physical activity level $(\mathrm{P}>0.1$ for both). Urine NTx levels did not differ between patients and reference subjects $(\mathrm{P}=0.451$; Table 5), between reference subjects and those with mild or moderate-to-severe eczema, or between those with mild and moderate-to-severe eczema $(\mathrm{P}>0.3$ for all). This biomarker showed an inverse correlation with subject's age $(r=-0.569, \mathrm{P}<0.001)$ but not BMI $\mathrm{Z}$-score $(r=-0.132, \mathrm{P}=0.101)$ or BMD Z-score at the radius $(r=-0.133, \mathrm{P}=0.097)$ or tibia $(r=-0.135$, $\mathrm{P}=0.093)$. Repeated analyses in eczema or reference subject subgroups yielded similar results.

Regarding the possible effects of calcium intake, the tertiles of daily intake were not associated with BMD Z-score at either the radius or tibia among reference subjects (Table 6). On the other hand, patients at the second tertile of calcium intake had a lower BMD Z-score at both the radius and tibia than those at the first and third tertiles $(\mathrm{P}=0.016$ and 0.015 , respectively by one-way ANOVA). Patients with the highest tertile of calcium intake had higher BMD Z-score at the tibia.

\section{Discussion}

This study is the first to report the effects of eczema and dietary intake on BMD in Chinese children in Hong Kong. Children with eczema had a higher intake of soybeans and miscellaneous dairy products but lower intake of eggs, beef, shellfish, vitamin D, calcium, and iron than the reference group. Despite these differences, children with eczema had similar BMD Z-scores at the radius and tibia and urinary NTx levels. We also observed a trend for patients with the highest tertile of calcium intake to have a higher BMD Z-score at the tibia.

Food allergy is commonly believed to be a major cause of eczema in the Chinese culture. Werfel and Breuer ${ }^{16}$ supported this by the finding that atopic dermatitis could be exacerbated by certain foods in more than $50 \%$ of affected children. Nonetheless, reactions induced by classic foods such as hen's eggs and cow's milk were less common in adolescents and adults than in young children. Food allergy in eczema may be immunoglobulin (Ig) E-mediated or nonIgE-mediated, thus food-induced eczema should be diagnosed only by thorough clinical history-taking and diagnostic work-up. Because of a possible co-existence of eczema and food allergy, dietary restrictions form an integral component of eczema management in children. A systematic review of 421 participants from nine randomised controlled trials only suggested some benefit of an egg-free diet in infants with suspected egg allergy who had positive specific IgE to eggs. No benefit, however, could be detected for the use of various exclusion diets in unselected people with atopic eczema. ${ }^{17}$ Clinicians should also bear in mind the dynamic nature of food allergy, and that young children might outgrow such allergies.

Eczema severity was associated with altered dietary intake in our children. Of Hong Kong children aged 2 to 7 years, $8 \%$ reported adverse food reactions, with the six leading foods being shellfish, eggs, peanuts, beef, cow's milk, and tree nuts. ${ }^{2}$ Such adverse reactions to multiple foods also led to worse quality of life. ${ }^{18}$ In the EuroPrevall study, shrimp, mango, milk, eggs, and peanuts were the foods most commonly reported to cause allergy among primary schoolchildren in Hong Kong. ${ }^{19,20}$ According to the presence of allergen-specific IgE, however, milk and egg sensitisation was identified in over $14 \%$ of these subjects whereas that for all other foods including shrimp and peanuts was less than $8 \%$. In our hospital-based patients, skin prick testing revealed that shellfish, peanuts, nuts, and eggs were the most 
common food allergens..$^{21}$ In general, allergy to milk, eggs, beef, and seafood is a common cultural belief in Chinese families with children having eczema. Consistently, our patients with moderate-to-severe eczema had a lower intake of eggs, beef, and shellfish as well as vitamin D, calcium, and iron (Tables 2 to 4). To compensate for such dietary restrictions, they ingested more soybeans and soybean products, as well as miscellaneous dairy products. These alterations were consistent with our earlier findings that dietary restrictions to avoid high calcium foods such as cow's milk were common among Hong Kong children with eczema. ${ }^{3,22}$ Milk intake was also negatively associated with eczema diagnosis among Spanish primary schoolchildren. ${ }^{23}$

Dietary intake of vitamin D was very low in our children, and virtually all (both cases and controls) ingested a lower amount than the age- and sexspecific dietary reference intake for the Chinese population (Table 4). Our data also suggested an inverse relationship between eczema severity and vitamin D intake. These results echoed those of our recent study in which low serum vitamin D level was highly prevalent in both Hong Kong children with eczema and non-allergic controls. ${ }^{24}$ Vitamin D deficiency was associated with disease severity in our eczema children. Our study found eczema severity to be associated with several single-nucleotide polymorphisms of vitamin D pathwaygenes. ${ }^{25}$ Besides its role in bone metabolism, vitamin D3 exerted pluripotent effects on both cutaneous adaptive (eg T-cell activation and dendritic cell maturation) and innate (eg expression of antimicrobial peptides) immunity. ${ }^{26,27}$ Our data suggested low serum levels of LL-37, the biologically active form of cathelicidin involved in antimicrobial defence, in children with eczema. ${ }^{28}$ Alterations in local vitamin D3 levels also modulated skin barrier function. ${ }^{29}$ Most children in Hong Kong, a subtropical region, had long school hours on weekdays and swam mainly in indoor pools. Thus, they are not expected to produce enough vitamin $\mathrm{D}$ from sunlight exposure. Together with our dietary data, we recommend that local children increase their outdoor activities and dietary intake of vitamin D.

Dietary intake of our children was recorded by FFQ. This method has been used to assess habitual intake over extended periods of time, ranging from the past month to the past year, by asking respondents to report frequency of consumption and often portion size for a defined list of foods and beverages. ${ }^{30}$ This allows for investigation of individual dietary pattern, ranking of usual individual intake, and examination of associations between frequency of consumption of certain items and individual clinical conditions. Woo et $\mathrm{al}^{9}$ developed an FFQ for the Hong Kong Chinese population that was later adapted and validated in local children and adolescents. ${ }^{11-13,18}$ Energy intake of two thirds of our subjects was below the Chinesespecific dietary reference intake (Table 4). Based on our data, FFQ would overestimate the intake of energy and macronutrients when compared with a 3-day food record. ${ }^{11}$ Thus, both our patients and controls had an inadequate nutritional intake.

Skeletal problems were common in children with food allergy and eczema. The BMD in children aged 8 to 17 years with CMA was markedly reduced for age, and calcium consumption was only one quarter that recommended. ${ }^{5}$ Beta-crosslaps concentration as a biomarker of bone turnover was lower in CMA patients than in controls. ${ }^{6}$ Osteoporosis and osteopenia were detected in 4.8\% and $32.8 \%$ of 125 adults with moderate-to-severe eczema, respectively. ${ }^{31}$ Low BMD in these patients did not seem to respond to calcium and/or vitamin D supplementation. ${ }^{32}$ Another small adult study found similar BMD between subjects with eczema and controls. ${ }^{33}$ In childhood eczema, patients with severe disease treated with topical corticosteroids and cyclosporin had much lower BMD as measured by dual energy X-ray absorptiometry (DEXA) than those prescribed topical corticosteroids alone. ${ }^{34}$ The BMD was similar between Dutch children with moderate-to-severe eczema and the general population. ${ }^{35}$ Overall, there are limited data regarding BMD in childhood eczema. In this study, children with eczema had similar sonographically measured BMD at the radius and tibia and urinary level of NTx when compared with controls (Table 5). Nonetheless, BMD Z-scores were lower among patients in the second than third tertile of dietary calcium intake (Table 6). We do not recommend unjustified restriction of calcium-rich foods such as cow's milk and soybean in children with eczema.

This study has several limitations. First, we did not record subjects' outdoor activities or measure their serum vitamin D level. Second, this study assessed subjects' dietary intake using a FFQ instead of a 3-day food record. The FFQ is cheap and easy to administer. Such FFQ with parental reporting was also a reasonably valid way to collect dietary data on children even in situations when parents do not observe all meals and snacks eaten by their child. ${ }^{36-38}$ Third, because of its cross-sectional nature, this study did not collect information about the duration of food avoidance. Fourth, BMD of our children was measured by ultrasound rather than DEXA; with the latter being the gold standard for diagnosing osteoporosis. There is substantial concern about radiation hazard especially in children. ${ }^{39}$ This study adopted the radiation-free technique of QUBS that was useful in assessing BMD in children..$^{40}$ Lastly, our patients with moderate-to-severe eczema were nearly 2 years older than the reference group although patients as a whole group were of similar age (Table 1). Because of the higher energy needs of 
older and bigger children, we adjusted for subjects' age and gender in multivariable regression analyses and compared their dietary intake with age- and gender-specific dietary reference intakes.

\section{Conclusion}

Dietary restrictions are common among Chinese children with eczema in Hong Kong. These patients had a lower calcium, vitamin D, and iron intake. Despite this, childhood eczema was not associated with diminished BMD. Nonetheless, a significant association was detected between calcium intake and BMD among these patients.

\section{Acknowledgements}

This work was funded by Direct Grants for Research (2011.1.058 and 2013.2.033) of the Chinese University of Hong Kong. We thank Ms Yvonne YF Ho and Ms Patty PP Tse for helping with patient assessment and data collection.

\section{Declaration}

The authors have disclosed no conflicts of interest.

\section{References}

1. Leung DY, Bieber T. Atopic dermatitis. Lancet 2003;361:151-60.

2. Leung TF, Yung E, Wong YS, Lam CW, Wong GW. Parentreported adverse food reactions in Hong Kong Chinese pre-schoolers: epidemiology, clinical spectrum and risk factors. Pediatr Allergy Immunol 2009;20:339-46.

3. Hon KL, Leung TF, Kam WY, Lam MC, Fok TF, Ng PC. Dietary restriction and supplementation in children with atopic eczema. Clin Exp Dermatol 2006;31:187-91.

4. Henderson RC, Hayes PR. Bone mineralization in children and adolescents with a milk allergy. Bone Miner 1994;27:112.

5. Jensen VB, Jørgensen IM, Rasmussen KB, Mølgaard C, Prahl P. Bone mineral status in children with cow milk allergy. Pediatr Allergy Immunol 2004;15:562-5.

6. Hidvégi E, Arató A, Cserháti E, Horváth C, Szabó A, Szabó A. Slight decrease in bone mineralization in cow milksensitive children. J Pediatr Gastroenterol Nutr 2003;36:449.

7. Hanifin JM, Rajka G. Diagnostic features of atopic dermatitis. Acta Derm (Stockh) 1980;92:44-7.

8. Hon KL, Leung TF, Wong Y, Fok TF. Lesson from performing SCORADs in children with atopic dermatitis: subjective symptoms do not correlate well with disease extent or intensity. Int J Dermatol 2006;45:728-30.

9. Woo J, Leung SS, Ho SC, Lam TH, Janus ED. A food frequency questionnaire for use in the Chinese population in Hong Kong: description and examination of validity. Nutr Res 1997;17:1633-41.

10. Chan RS, Woo J, Chan DC, Cheung CS, Lo DH. Estimated net endogenous acid production and intake of bone healthrelated nutrients in Hong Kong Chinese adolescents. Eur J Clin Nutr 2009;63:505-12.

11. Kwok FY, Ho YY, Chow CM, So CY, Leung TF. Assessment of nutrient intakes of picky-eating Chinese preschoolers using a modified food frequency questionnaire. World J Pediatr 2013;9:58-63.

12. Yu CC, Sung RY, Hau KT, Lam PK, Nelson EA, So RC. The effect of diet and strength training on obese children's physical self-concept. J Sports Med Phys Fitness 2008;48:76-82.

13. Bollen AM, Eyre DR. Bone resorption rates in children monitored by the urinary assay of collagen type 1 crosslinked peptides. Bone 1994;15:31-4.

14. Jones G, Boon P. Which bone mass measures discriminate adolescents who have fractured from those who have not? Osteoporosis Int 2008;19:251-5.

15. Christoforidis A, Printza N, Gkogka C, et al. Comparative study of quantitative ultrasonography and dual-energy $\mathrm{X}$-ray absorptiometry for evaluating renal osteodystrophy in children with chronic kidney disease. J Bone Miner Metab 2011;29:321-7.

16. Werfel T, Breuer K. Role of food allergy in atopic dermatitis. Curr Opin Allergy Clin Immunol 2004;4:379-85.

17. Bath-Hextall F, Delamere FM, Williams HC. Dietary exclusions for improving established atopic eczema in adults and children: systematic review. Allergy 2009;64:25864 .

18. Leung TF, Yung E, Wong YS, Li CY, Wong GW. Qualityof-life assessment in Chinese families with food-allergic children. Clin Exp Allergy 2009;39:890-6.

19. Wong GW, Mahesh PA, Ogorodova L, et al. The EuroPrevall-INCO surveys on the prevalence of food allergies in children from China, India and Russia: the study methodology. Allergy 2010;65:385-90.

20. Wong GW, Ogorodova L, Mahesh PA, et al. Food allergy in schoolchildren from China, Russia and India: the EuroPrevall-INCO surveys. Allergy 2011;66 Suppl 94:27.

21. Hon KL, Wang SS, Wong WL, Poon WK, Mak KY, Leung TF. Skin prick testing in atopic eczema: atopic to what and at what age? World J Pediatr 2012;8:164-8.

22. Hon KL, Leung TF, Lam MC, et al. Eczema exacerbation and food atopy beyond infancy: how should we advise Chinese parents about dietary history, eczema severity and skin prick testing? Adv Ther 2007;24:223-30.

23. Suárez-Varela MM, Alvarez LG, Kogan MD, et al. Diet and prevalence of atopic eczema in 6 to 7-year-old schoolchildren in Spain: ISAAC phase III. J Investig Allergol Clin Immunol 2010;20:469-75.

24. Wang SS, Hon KL, Kong AP, Pong HN, Wong GW, Leung TF. Vitamin D deficiency is associated with diagnosis and severity of childhood atopic dermatitis. Pediatr Allergy Immunol 2014;25:30-5.

25. Wang SS, Hon KL, Kong AP, et al. Eczema phenotypes are associated with multiple vitamin D pathway genes in Chinese children. Allergy 2014;69:118-24.

26. Bikle DD. What is new in vitamin D: 2006-2007. Curr Opin Rheumatol 2007;19:383-8.

27. Schauber J, Dorschner RA, Yamasaki K, Brouha B, Gallo RL. Control of the innate epithelial antimicrobial response is cell-type specific and dependent on relevant microenvironmental stimuli. Immunology 2006;118:50919.

28. Leung TF, Ching KW, Kong AP, Wong GW, Chan JC, Hon KL. Circulating LL-37 is a biomarker for eczema severity in children. J Eur Acad Dermatol Venereol 2012;26:518-22. 29. Bikle DD, Chang S, Crumrine D, et al. Mice lacking 25OHD 
1alpha-hydroxylase demonstrate decreased epidermal differentiation and barrier function. J Steroid Biochem Mol Biol 2004;89-90(1-5):347-53.

30. Thompson FE, Byers T. Dietary assessment resource manual. J Nutr 1994;124(11 Suppl):2245S-2317S.

31. Haeck IM, Hamdy NA, Timmer-de Mik L, et al. Low bone mineral density in adult patients with moderate to severe atopic dermatitis. Br J Dermatol 2009;161:1248-54.

32. Haeck I, van Velsen S, de Bruin-Weller M, BruijnzeelKoomen C. Bone mineral density in patients with atopic dermatitis. Chem Immunol Allergy 2012;96:96-9.

33. Aalto-Korte K, Turpeinen M. Bone mineral density in patients with atopic dermatitis. $\mathrm{Br} \mathrm{J}$ Dermatol 1997;136:172-5.

34. Pedreira CC, King E, Jones G, et al. Oral cyclosporin plus topical corticosteroid therapy diminishes bone mass in children with eczema. Pediatr Dermatol 2007;24:613-20.

35. van Velsen SG, Knol MJ, van Eijk RL, et al. Bone mineral density in children with moderate to severe atopic dermatitis. J Am Acad Dermatol 2010;63:824-31.
36. Andersen LF, Lande B, Trygg K, Hay G. Validation of a semi-quantitative food-frequency questionnaire used among 2-year-old Norwegian children. Public Health Nutr 2004;7:757-64.

37. Blum RE, Wei EK, Rockett HR, et al. Validation of a food frequency questionnaire in native American and Caucasian children 1 to 5 years of age. Matern Child Health J 1999;3:167-72.

38. Parrish LA, Marshall JA, Krebs NF, Rewers M, Norris JM. Validation of a food frequency questionnaire in preschool children. Epidemiology 2003;14:213-7.

39. Pearce MS, Salotti JA, Little MP, et al. Radiation exposure from CT scans in childhood and subsequent risk of leukaemia and brain tumours: a retrospective cohort study. Lancet 2012;380:499-505.

40. Mainz JG, Sauner D, Malich A, et al. Cross-sectional study on bone density-related sonographic parameters in children with asthma: correlation to therapy with inhaled corticosteroids and disease severity. J Bone Miner Metab 2008;26:485-92. 